\title{
On the Piatetski-Shapiro-Vinogradov Theorem
}

\author{
by \\ Chaohua JiA (Beijing)
}

1. Introduction. In 1937, I. M. Vinogradov proved the famous three primes theorem. It states that for every sufficiently large odd integer $N$,

$$
\sum_{N=p_{1}+p_{2}+p_{3}} 1=\frac{1}{2}(1+o(1)) C(N) \frac{N^{2}}{\log ^{3} N},
$$

where

$$
C(N)=\prod_{p \mid N}\left(1-\frac{1}{(p-1)^{2}}\right) \prod_{p \nmid N}\left(1+\frac{1}{(p-1)^{3}}\right) .
$$

Afterwards people have been looking for thin subsets of primes for which the three primes theorem still holds. In 1986, Wirsing [13] showed that there exists such a set $S$ with the property that

$$
\sum_{\substack{p \leq x \\ p \in S}} 1 \ll(x \log x)^{1 / 3} .
$$

It is also interesting to find more familiar thin sets of primes which serve this purpose. An example is the set of Piatetski-Shapiro primes of type $\gamma$ which are of the form $\left[n^{1 / \gamma}\right]$. We denote this set by $P_{\gamma}$.

For the counting function of $P_{\gamma}$, Piatetski-Shapiro [11] first showed that for $11 / 12<\gamma \leq 1$ (the case $\gamma>1$ is trivial),

$$
P_{\gamma}(x)=\sum_{\substack{p \leq x \\ p=\left[n^{1 / \gamma}\right]}} 1=(1+o(1)) \frac{x^{\gamma}}{\log x} .
$$

Heath-Brown [4] extended the range to $662 / 755<\gamma \leq 1$. Further improvements were made by Kolesnik [8], Liu and Rivat [9].

Project supported by the Tian Yuan Item in the National Natural Science Foundation of China. 
Jia Chaohua [7] was the first to apply the sieve method to the investigation of Piatetski-Shapiro primes and proved that, for $17 / 20<\gamma \leq 1$ and sufficiently large $x$,

$$
P_{\gamma}(x) \geq \frac{\varrho_{0} x^{\gamma}}{\log x},
$$

where $\varrho_{0}$ is a definite positive constant. Using the sieve method of Harman [3], Jia Chaohua [6] extended the range in (4) to $11 / 13<\gamma \leq 1$.

Now we come back to the subject of this paper. In 1992, Balog and Friedlander [1] proved the Piatetski-Shapiro-Vinogradov theorem: If $\gamma_{1}, \gamma_{2}$, $\gamma_{3}$ are fixed subject to $0<\gamma_{i} \leq 1$ and $9\left(1-\gamma_{3}\right)<1,9\left(1-\gamma_{2}\right)+6\left(1-\gamma_{3}\right)<1$, $9\left(1-\gamma_{1}\right)+6\left(1-\gamma_{2}\right)+6\left(1-\gamma_{3}\right)<1$, then for every sufficiently large odd integer $N$,

$$
\begin{aligned}
T(N) & =\sum_{\substack{N=p_{1}+p_{2}+p_{3} \\
p_{i} \in P_{\gamma_{i}}}} 1 \\
& =(1+o(1)) \frac{\gamma_{1} \gamma_{2} \gamma_{3} \Gamma\left(\gamma_{1}\right) \Gamma\left(\gamma_{2}\right) \Gamma\left(\gamma_{3}\right)}{\Gamma\left(\gamma_{1}+\gamma_{2}+\gamma_{3}\right)} \cdot \frac{C(N) N^{\gamma_{1}+\gamma_{2}+\gamma_{3}-1}}{\log ^{3} N} .
\end{aligned}
$$

From this theorem, there are two interesting corollaries:

COROllary 1. For any fixed $20 / 21<\gamma \leq 1$, every sufficiently large odd integer $N$ can be written as a sum of three Piatetski-Shapiro primes of type $\gamma$.

COROLlary 2. For any fixed $8 / 9<\gamma \leq 1$, every sufficiently large odd integer $N$ can be written as a sum of one Piatetski-Shapiro prime of type $\gamma$ and two primes.

In his doctoral thesis, J. Rivat extended the range $20 / 21<\gamma \leq 1$ in Corollary 1 to $188 / 199<\gamma \leq 1$.

In this paper, we shall apply the sieve method combined with the circle method to this problem and prove:

TheOREM. If $\gamma$ is fixed with $15 / 16<\gamma \leq 1$, then for every sufficiently large odd integer $N$,

$$
T_{1}(N)=\sum_{\substack{N=p_{1}+p_{2}+p_{3} \\ p_{i} \in P_{\gamma}}} 1 \geq \frac{\varrho_{0} C(N) N^{3 \gamma-1}}{\log ^{3} N},
$$

where $\varrho_{0}$ is a definite positive constant.

Our Theorem improves Corollary 1 of Balog and Friedlander.

Throughout this paper, we always assume that $N$ is a sufficiently large odd integer and $\varepsilon$ is a sufficiently small positive constant. Assume that $c$, $c_{1}, c_{2}$ are positive constants which have different values at different places. 
$m \sim M$ means that there are positive constants $c_{1}$ and $c_{2}$ such that $c_{1} M<$ $m \leq c_{2} M$. We also assume that $\gamma$ is fixed with $15 / 16<\gamma \leq 20 / 21$ and that

$$
N(d)=\left[-d^{\gamma}\right]-\left[-(d+1)^{\gamma}\right] .
$$

The author would like to thank Profs. Wang Yuan and Pan Chengbiao for their encouragement. He also thanks the referee for the information about Rivat's thesis.

2. Some preliminary lemmas. In the following, we assume that

$$
H=N^{1-\gamma+\Delta+8 \varepsilon} \text {. }
$$

By the discussion in [1], the asymptotic formula that for $0 \leq \Delta \leq 1-\gamma$,

$$
\sum_{N / 10<p \leq N} N(p) e(\alpha p)=\gamma \sum_{N / 10<p \leq N} p^{\gamma-1} e(\alpha p)+O\left(N^{\gamma-\Delta-5 \varepsilon}\right),
$$

depends on the fact that for $J \leq H$ and $0 \leq u \leq 1$,

$$
\min \left(1, \frac{N^{1-\gamma}}{J}\right) \sum_{h \sim J}\left|\sum_{n \sim N} \Lambda(n) e\left(\alpha n+h(n+u)^{\gamma}\right)\right| \ll N^{1-\Delta-6 \varepsilon} .
$$

Lemma 1. Assume that $N^{1-\gamma+2 \Delta+30 \varepsilon} \ll M \ll N^{5 \gamma-4-6 \Delta-120 \varepsilon}$ and that $a(m), b(k)=O(1)$. Then for $J \leq H$ and $0 \leq u \leq 1$, we have

$$
\begin{array}{r}
\min \left(1, \frac{N^{1-\gamma}}{J}\right) \sum_{h \sim J}\left|\sum_{m \sim M} \sum_{k m \sim N} a(m) b(k) e\left(\alpha k m+h(k m+u)^{\gamma}\right)\right| \\
\ll N^{1-\Delta-10 \varepsilon .}
\end{array}
$$

This is Proposition 2 of [1].

Lemma 2. Assume that $M \ll N^{4 \gamma-3-5 \Delta-50 \varepsilon}, a(m)=O(1)$ and

$$
6(1-\gamma)+\frac{19}{3} \Delta<1
$$

Then for $J \leq H$ and $0 \leq u \leq 1$, we have

$\min \left(1, \frac{N^{1-\gamma}}{J}\right) \sum_{h \sim J}\left|\sum_{m \sim M} a(m) \sum_{k m \sim N} e\left(\alpha k m+h(k m+u)^{\gamma}\right)\right| \ll N^{1-\Delta-10 \varepsilon}$.

This is Proposition 3 of [1].

Lemma 3. Assume that $a(m), b(k)=O(1)$ and that for $V \ll M \ll N / V$,

$$
\sum_{m \sim M} \sum_{k m \sim N} a(m) b(k) F(k m) \ll \Phi N^{-10 \varepsilon},
$$

and for $M \ll V^{2}$,

$$
\sum_{m \sim M} a(m) \sum_{k m \sim N} F(k m) \ll \Phi N^{-10 \varepsilon} .
$$


Then

$$
\sum_{n \sim N} \Lambda(n) F(n) \ll \Phi N^{-6 \varepsilon} .
$$

This can be deduced from Vaughan's identity.

LEMMA 4. Under the above assumptions, we have

$$
\sum_{N / 10<p \leq N} N(p) e(\alpha p)=\gamma \sum_{N / 10<p \leq N} p^{\gamma-1} e(\alpha p)+O\left(N^{3 \gamma / 2-1 / 2-5 \varepsilon}\right) .
$$

Proof. In order to prove (12), we should prove (10) with $\Delta=\frac{1}{2}(1-\gamma)$. Now,

$$
\begin{aligned}
\min (1, & \left.\frac{N^{1-\gamma}}{J}\right) \sum_{h \sim J}\left|\sum_{n \sim N} \Lambda(n) e\left(\alpha n+h(n+u)^{\gamma}\right)\right| \\
& =\min \left(1, \frac{N^{1-\gamma}}{J}\right) \sum_{h \sim J} \varepsilon(h, \alpha, u) \sum_{n \sim N} \Lambda(n) e\left(\alpha n+h(n+u)^{\gamma}\right) .
\end{aligned}
$$

Let $V=N^{2(1-\gamma)+30 \varepsilon}$ and

$$
F(n)=\min \left(1, \frac{N^{1-\gamma}}{J}\right) \sum_{h \sim J} \varepsilon(h, \alpha, u) e\left(\alpha n+h(n+u)^{\gamma}\right) .
$$

Note that (11) is satisfied for $0 \leq \Delta \leq 1-\gamma$. By Lemmas 1-3, we can get

$$
\sum_{n \sim N} \Lambda(n) F(n) \ll N^{3 \gamma / 2-1 / 2-6 \varepsilon} .
$$

So Lemma 4 follows.

Lemma 5. Assume that $|\alpha-a / q|<1 / q^{2},(a, q)=1$. Then

$$
\sum_{p \sim N} e(\alpha p) \ll\left(\frac{N}{\sqrt{q}}+\sqrt{N q}+N^{4 / 5}\right) \log ^{5} N .
$$

We refer to Section 25 of [2].

LEMma 6. Let

$$
d_{r}(n)=\sum_{n=n_{1} \ldots n_{r}} 1
$$

and $k$ be a positive integer. Then

$$
\sum_{n \leq x} d_{r}^{k}(n) \ll x(\log x)^{r^{k}-1}
$$

See Theorem 2 of [12]. 
LEMMA 7. Let

$$
C(q, m)=\sum_{\substack{r=1 \\(r, q)=1}}^{q} e\left(\frac{r m}{q}\right) .
$$

Then $C(q, m)$ is a multiplicative function of $q$ and

$$
C(q, m)=\mu\left(\frac{q}{(q, m)}\right) \frac{\varphi(q)}{\varphi\left(\frac{q}{(m, q)}\right)} .
$$

See Lemma 1.2 of [10].

We define $w(u)$ as the continuous solution of the equations

$$
\begin{gathered}
w(u)=1 / u, \quad 1 \leq u \leq 2, \\
(u w(u))^{\prime}=w(u-1), \quad u>2 .
\end{gathered}
$$

$w(u)$ is called Buchstab's function; it plays an important role in finding asymptotic formulas in the sieve method. In particular,

$$
w(u)= \begin{cases}\frac{1+\log (u-1)}{u}, & 2 \leq u \leq 3 \\ \frac{1+\log (u-1)}{u}+\frac{1}{u} \int_{2}^{u-1} \frac{\log (t-1)}{t} d t, & 3 \leq u \leq 4 .\end{cases}
$$

LEMma 8. We have the following bounds:

(i) $w(u) \geq 0.5607$ for $u \geq 2.47$;

(ii) $w(u) \leq 0.5644$ for $u \geq 3$;

(iii) $w(u) \leq 0.5672$ for $u \geq 1.7631$;

(iv) $w(u) \geq 0.5$ for $u \geq 1$.

Proof. (i) First assume $3 \leq u \leq 4$. By (15), we have

$$
w^{\prime}(u)=\frac{w(u-1)-w(u)}{u}=\frac{1}{u^{2}}(u w(u-1)-u w(u)) .
$$

Now we investigate the behaviour of the function

$$
h(u)=u w(u-1)-u w(u)
$$

We have

$$
\begin{aligned}
h^{\prime}(u) & =((u-1) w(u-1))^{\prime}-(u w(u))^{\prime}+w^{\prime}(u-1) \\
& =w(u-2)-w(u-1)+\frac{w(u-2)-w(u-1)}{u-1} \\
& =\frac{u(1-(u-2) \log (u-2))}{(u-2)(u-1)^{2}} .
\end{aligned}
$$

There is exactly one $u_{0}$ satisfying

$$
1-\left(u_{0}-2\right) \log \left(u_{0}-2\right)=0
$$

Calculation shows $3.7632 \leq u_{0} \leq 3.7633$. 
We have $h^{\prime}(u)>0$ to the left of $u_{0}$, and $h^{\prime}(u)<0$ to the right of $u_{0}$. Consequently, if $u_{0} \leq u \leq 4$, then $h(u) \geq h(4)=4(w(3)-w(4))>0$. Note that $h(3)=3(w(2)-w(3))<0$ and that $h(u)$ is an increasing function in the interval $\left[3, u_{0}\right]$. So, $h(u)$ has exactly one zero $v_{0}$ in the interval $[3,4]$. Calculation shows $3.469 \leq v_{0} \leq 3.47$. We have $h(u)<0$ to the left of $v_{0}$, and $h(u)>0$ to the right of $v_{0}$. The same holds for $w^{\prime}(u)$. Hence, $w(u) \geq w\left(v_{0}\right)$.

We note that for $3 \leq u \leq 4$,

$$
\left(\frac{1+\log (u-1)}{u}\right)^{\prime}=\frac{1-(u-1) \log (u-1)}{(u-1) u^{2}}<0 .
$$

The fact that for $2 \leq t \leq 3$,

$$
\left(\frac{\log (t-1)}{t}\right)^{\prime}=\frac{t-(t-1) \log (t-1)}{(t-1) t^{2}} \geq 0,
$$

implies that for $3 \leq u \leq 4$,

$$
\begin{aligned}
\left(\frac{1}{u} \int_{2}^{u-1} \frac{\log (t-1)}{t} d t\right)^{\prime} & =\frac{1}{u^{2}}\left(\frac{u \log (u-2)}{u-1}-\int_{2}^{u-1} \frac{\log (t-1)}{t} d t\right) \\
& \geq \frac{1}{u^{2}}\left(\frac{u \log (u-2)}{u-1}-\frac{(u-3) \log (u-2)}{u-1}\right) \geq 0 .
\end{aligned}
$$

Therefore we have

$$
w\left(v_{0}\right) \geq \frac{1+\log (2.47)}{3.47}+\frac{1}{3.469} \int_{2}^{2.469} \frac{\log (t-1)}{t} d t \geq 0.5607 .
$$

From the above discussion, we get $w(u) \geq 0.5607$ for $3 \leq u \leq 4$.

Then we employ induction. Suppose that $w(u) \geq 0.5607$ for $3 \leq k \leq u \leq$ $k+1$. For $k+1 \leq u \leq k+2$, we have

$$
u w(u)=(k+1) w(k+1)+\int_{k}^{u-1} w(t) d t \geq 0.5607 u .
$$

By induction, we conclude that $w(u) \geq 0.5607$ for $u \geq 3$.

Now we turn to the case $2 \leq u \leq 3$. Then

$$
w^{\prime}(u)=\frac{1-(u-1) \log (u-1)}{(u-1) u^{2}}
$$

has exactly one zero $z_{0}$ with $2.7632 \leq z_{0} \leq 2.7633$.

We have $w^{\prime}(u)>0$ to the left of $z_{0}$, and $w^{\prime}(u)<0$ to the right of $z_{0}$. Therefore $w(u) \geq \min (w(2.47), w(3)) \geq 0.5607$ for $2.47 \leq u \leq 3$, and $w(u) \leq w\left(z_{0}\right) \leq(1+\log (1.7633)) / 2.7632 \leq 0.5672$ for $2 \leq u \leq 3$.

(ii) The discussion in (i) implies that $w(u) \leq \max (w(3), w(4)) \leq 0.5644$ for $3 \leq u \leq 4$. By induction, it follows that $w(u) \leq 0.5644$ for $u \geq 3$. 
(iii) The discussion in (i) shows that $w(u) \leq 0.5672$ for $2 \leq u \leq 3$. For $1.7631 \leq u \leq 2$, we have $w(u)=1 / u \leq 0.5672$.

(iv) It is easy to see that $w(u)=1 / u \geq 0.5$ for $1 \leq u \leq 2$. By induction, we get the same conclusion for all $u \geq 1$.

The proof of Lemma 8 is complete.

Lemma 9. Assume that $\mathcal{E}=\{n: x<n \leq 2 x\}$ and that $z \leq x$. Let

$$
P(z)=\prod_{p<z} p .
$$

Then for sufficiently large $x$ and $z$, we have

$$
S(\mathcal{E}, z)=\sum_{\substack{x<n \leq 2 x \\(n, P(z))=1}} 1=\left(w\left(\frac{\log x}{\log z}\right)+O(\varepsilon)\right) \frac{x}{\log z} .
$$

We refer to Lemma 5 of [6]. When $(2 x)^{1 / 2}<z \leq x$, this is the prime number theorem.

\section{Mean value formulas in the sieve method}

Lemma 10. Assume that $M, K \ll N^{5 / 16}$ and that $a(m), b(k)=O(1)$. Let

$$
I(N)=\sum_{\substack{N=n_{1}+n_{2}+n_{3} \\ N / 10<n_{1}, n_{2}, n_{3} \leq N}} \frac{\gamma^{3}\left(n_{1} n_{2} n_{3}\right)^{\gamma-1}}{\log n_{2} \log n_{3}}
$$

and

$$
\omega(r)=\prod_{\substack{p|r \\ p| N}}\left(1+\frac{1}{p-1}\right) \prod_{\substack{p \mid r \\ p \nmid N}}\left(1-\frac{1}{(p-1)^{2}}\right) .
$$

Then

$$
\begin{aligned}
\sum_{m \sim M} \sum_{k \sim K} a(m) b(k)\left(\sum_{\substack{N=m k l+p_{2}+p_{3} \\
N / 10<m k l \leq N \\
N / 10<p_{2}, p_{3} \leq N}} N(m k l) N\left(p_{2}\right) N\left(p_{3}\right)-\frac{\omega(m k)}{m k} I(N)\right) \\
=O\left(\frac{N^{3 \gamma-1}}{\log ^{20} N}\right) .
\end{aligned}
$$

Proof. We have

$$
\Sigma_{1}=\sum_{m \sim M} \sum_{k \sim K} a(m) b(k) \sum_{\substack{N=m k l+p_{2}+p_{3} \\ N / 10<m k l \leq N \\ N / 10<p_{2}, p_{3} \leq N}} N(m k l) N\left(p_{2}\right) N\left(p_{3}\right)
$$




$$
\begin{aligned}
= & \int_{0}^{1} \sum_{\substack{N / 10<m k l \leq N \\
m \sim M, k \sim K}} a(m) b(k) N(m k l) e(\alpha m k l) \\
& \times\left(\sum_{N / 10<p \leq N} N(p) e(\alpha p)\right)^{2} e(-\alpha N) d \alpha .
\end{aligned}
$$

Let

$$
\begin{aligned}
& g(\alpha)=\sum_{\substack{N / 10<m k l \leq N \\
m \sim M, k \sim K}} a(m) b(k) N(m k l) e(\alpha m k l), \\
& f(\alpha)=\gamma \sum_{\substack{N / 10<m k l \leq N \\
m \sim M, k \sim K}} a(m) b(k)(m k l)^{\gamma-1} e(\alpha m k l) .
\end{aligned}
$$

By the discussion in [1], the asymptotic formula

$$
g(\alpha)=f(\alpha)+O\left(N^{2 \gamma-1-5 \varepsilon}\right)
$$

depends on the fact that for $J \leq H_{1}=N^{2(1-\gamma)+8 \varepsilon}$ and $0 \leq u \leq 1$,

(23) $\Sigma_{2}=\min \left(1, \frac{N^{1-\gamma}}{J}\right)$

$$
\begin{aligned}
& \quad \times \sum_{h \sim J}\left|\sum_{m \sim M} \sum_{k \sim K} \sum_{m k l \sim N} a(m) b(k) e\left(\alpha m k l+h(m k l+u)^{\gamma}\right)\right| \\
& \ll N^{\gamma-6 \varepsilon} .
\end{aligned}
$$

If either $M$ or $K$ is larger than $N^{3 / 16}$, then by Lemma 1 with $\Delta=$ $1-\gamma$, we obtain $\Sigma_{2} \ll N^{\gamma-10 \varepsilon}$. If $M, K \leq N^{3 / 16}$, then $K M \ll N^{6 / 16} \ll$ $N^{9 \gamma-8-50 \varepsilon}$. By Lemma 2 with $\Delta=1-\gamma$, we also get $\Sigma_{2} \ll N^{\gamma-10 \varepsilon}$. Hence, (22) holds.

Let

$$
D(\alpha)=\sum_{N / 10<p \leq N} N(p) e(\alpha p), \quad S(\alpha)=\gamma \sum_{N / 10<p \leq N} p^{\gamma-1} e(\alpha p) .
$$

From (12) and (22), it follows that

$$
\begin{aligned}
g(\alpha) D^{2}(\alpha)-f(\alpha) S^{2}(\alpha)= & (g(\alpha)-f(\alpha)) D^{2}(\alpha)+f(\alpha)(D(\alpha)-S(\alpha)) D(\alpha) \\
& +f(\alpha) S(\alpha)(D(\alpha)-S(\alpha)) \\
\ll & N^{2 \gamma-1-5 \varepsilon}|D(\alpha)|^{2}+N^{3 \gamma / 2-1 / 2-5 \varepsilon}|f(\alpha) D(\alpha)| \\
& +N^{3 \gamma / 2-1 / 2-5 \varepsilon}|f(\alpha) S(\alpha)| .
\end{aligned}
$$

Thus

$$
\Sigma_{1}=\int_{0}^{1} g(\alpha) D^{2}(\alpha) e(-\alpha N) d \alpha=\int_{0}^{1} f(\alpha) S^{2}(\alpha) e(-\alpha N) d \alpha+\Psi,
$$


where

(24) $\Psi \ll N^{2 \gamma-1-5 \varepsilon} \int_{0}^{1}|D(\alpha)|^{2} d \alpha+N^{3 \gamma / 2-1 / 2-5 \varepsilon}\left(\int_{0}^{1}|f(\alpha)|^{2} d \alpha\right)^{1 / 2}$

$$
\begin{aligned}
& \times\left(\int_{0}^{1}|D(\alpha)|^{2} d \alpha\right)^{1 / 2}+N^{3 \gamma / 2-1 / 2-5 \varepsilon}\left(\int_{0}^{1}|f(\alpha)|^{2} d \alpha\right)^{1 / 2} \\
& \times\left(\int_{0}^{1}|S(\alpha)|^{2} d \alpha\right)^{1 / 2} \\
& \ll N^{3 \gamma-1-4 \varepsilon}
\end{aligned}
$$

where we note that $N(p)=0$ or 1 and that $p \in P_{\gamma}$ is equivalent to $N(p)=1$; we also use the estimation $\sum_{p \leq N} N(p) \leq \sum_{n \leq N} N(n) \ll N^{\gamma}$.

In the following we investigate

$$
\Sigma_{3}=\int_{0}^{1} f(\alpha) S^{2}(\alpha) e(-\alpha N) d \alpha .
$$

Let $Q=N \log ^{-80} N$. We divide the interval $[-1 / Q, 1-1 / Q)$ into two parts:

$$
\begin{gathered}
E_{1}=\left\{\alpha: \alpha \in[-1 / Q, 1-1 / Q), \alpha=a / q+\beta, q \leq \log ^{80} N,\right. \\
0 \leq a \leq q-1,(a, q)=1,|\beta| \leq 1 /(q Q)\}, \\
E_{2}=[-1 / Q, 1-1 / Q)-E_{1} .
\end{gathered}
$$

Then

$$
\Sigma_{3}=\left(\int_{E_{1}}+\int_{E_{2}}\right) f(\alpha) S^{2}(\alpha) e(-\alpha N) d \alpha .
$$

For any $\alpha \in E_{2}$, there is one $q\left(\log ^{80} N<q \leq Q\right)$ such that $|\alpha-a / q|<$ $1 /(q Q)$. Lemma 5 yields $S(\alpha) \ll N^{\gamma} \log ^{-35} N$. Hence,

$$
\begin{aligned}
\int_{E_{2}} f(\alpha) S^{2}(\alpha) e(-\alpha N) d \alpha & \\
& \ll \frac{N^{\gamma}}{\log ^{35} N}\left(\int_{0}^{1}|f(\alpha)|^{2} d \alpha\right)^{1 / 2}\left(\int_{0}^{1}|S(\alpha)|^{2} d \alpha\right)^{1 / 2} \ll \frac{N^{3 \gamma-1}}{\log ^{20} N} .
\end{aligned}
$$

If $\alpha=a / q+\beta \in E_{1}$, then

$$
f(\alpha)=\sum_{\substack{N / 10<r l \leq N \\ r \sim R}} j(r)(r l)^{\gamma-1} e(\alpha r l),
$$

where $R=M K$ and

$$
j(r)=\gamma \sum_{\substack{m k=r \\ m \sim M, k \sim K}} a(m) b(k) .
$$


If $q \nmid b$, then

$$
\sum_{l \leq x} e(b l / q)=O(q) .
$$

From this and partial summation, we know that

$$
\begin{aligned}
\sum_{\substack{N / 10<r l \leq N \\
r \sim R, q \nmid r}} j(r)(r l)^{\gamma-1} e(\alpha r l) & \\
& =\sum_{\substack{r \sim R \\
q \nmid r}} j(r) r^{\gamma-1} \sum_{N /(10 r)<l \leq N / r} l^{\gamma-1} e\left(\frac{\operatorname{arl}}{q}+\beta r l\right) \\
& \ll N^{\gamma-1} \sum_{r \sim R} d(r) \log ^{80} N \ll N^{\gamma-\varepsilon} .
\end{aligned}
$$

On the other hand,

$$
\sum_{\substack{N / 10<r l \leq N \\ r \sim R, q \mid r}} j(r)(r l)^{\gamma-1} e(\alpha r l)=\sum_{\substack{r \sim R \\ q \mid r}} j(r) r^{\gamma-1} \sum_{N /(10 r)<l \leq N / r} l^{\gamma-1} e(\beta r l) .
$$

Now,

$$
\begin{aligned}
\sum_{N /(10 r)<l \leq N / r} l^{\gamma-1} e(\beta r l) \\
=\int_{N /(10 r)}^{N / r} t^{\gamma-1} e(\beta r t) d[t] \\
=\int_{N /(10 r)}^{N / r} t^{\gamma-1} e(\beta r t) d t-\int_{N /(10 r)}^{N / r} t^{\gamma-1} e(\beta r t) d(\{t\}) \\
=\frac{1}{r^{\gamma}} \int_{N / 10}^{N} u^{\gamma-1} e(\beta u) d u+O\left(\left(\frac{N}{r}\right)^{\gamma-1} \log ^{80} N\right) \\
=\frac{1}{r^{\gamma}} \sum_{N / 10<s \leq N} s^{\gamma-1} e(\beta s)+O\left(\left(\frac{N}{r}\right)^{\gamma-1} \log ^{80} N\right) .
\end{aligned}
$$

Thus,

$$
f(\alpha)=\sum_{\substack{r \sim R \\ q \mid r}} \frac{j(r)}{r} \sum_{N / 10<s \leq N} s^{\gamma-1} e(\beta s)+O\left(N^{\gamma-\varepsilon}\right) .
$$

The prime number theorem for arithmetic progressions (refer to Section 22 of [2]) yields that, for $q \leq \log ^{80} N,(l, q)=1$ and $N / 10<t \leq N$, 
(26) $\pi(t ; l, q)=\sum_{\substack{N / 10<p \leq t \\ p \equiv l(\bmod q)}} 1=\frac{1}{\varphi(q)} \int_{N / 10}^{t} \frac{d u}{\log u}+O(N \exp (-c \sqrt{\log N}))$.

Now,

$$
\sum_{N / 10<p \leq N} p^{\gamma-1} e(\alpha p)=\sum_{\substack{l=1 \\(l, q)=1}}^{q} e\left(\frac{a l}{q}\right) \sum_{\substack{N / 10<p \leq N \\ p \equiv l(\bmod q)}} p^{\gamma-1} e(\beta p),
$$

and so

$$
\begin{aligned}
\sum_{\substack{N / 10<p \leq N \\
p \equiv l(\bmod q)}} p^{\gamma-1} e(\beta p)= & \int_{N / 10}^{N} t^{\gamma-1} e(\beta t) d(\pi(t ; l, q)) \\
= & \frac{1}{\varphi(q)} \int_{N / 10}^{N} \frac{t^{\gamma-1} e(\beta t)}{\log t} d t \\
& +O\left(N^{\gamma} \exp \left(-c_{1} \sqrt{\log N}\right)\right) \\
= & \frac{1}{\varphi(q)} \sum_{N / 10<s \leq N} \frac{s^{\gamma-1} e(\beta s)}{\log s} \\
& +O\left(N^{\gamma} \exp \left(-c_{1} \sqrt{\log N}\right)\right) .
\end{aligned}
$$

Combining the above discussion with Lemma 7 , we have

$$
\begin{aligned}
\sum_{N / 10<p \leq N} p^{\gamma-1} e(\alpha p)= & \frac{\mu(q)}{\varphi(q)} \sum_{N / 10<s \leq N} \frac{s^{\gamma-1} e(\beta s)}{\log s} \\
& +O\left(N^{\gamma} \exp \left(-c_{2} \sqrt{\log N}\right)\right) .
\end{aligned}
$$

Altogether, we get

$$
\begin{aligned}
\Sigma_{4}= & \int_{E_{1}} f(\alpha) S^{2}(\alpha) e(-\alpha N) d \alpha \\
= & \sum_{q \leq \log ^{80}} \sum_{N}^{q-1} e\left(-\frac{a N}{q}\right) \int_{-1 /(q Q)}^{1 /(q Q)}(a, q)=1 \\
= & \gamma^{2} \sum_{q \leq \log ^{80} N} \frac{\mu^{2}(q) C(q,-N)}{\varphi^{2}(q)} \sum_{r \sim R} \frac{j(r)}{r} \int_{-1 /(q Q)}^{1 /(q Q)}\left(\sum_{N / 10<s \leq N} s^{\gamma-1} e(\beta s)\right) \\
& \times\left(\sum_{N / 10<s \leq N} \frac{s^{\gamma-1} e(\beta s)}{\log s}\right)^{2} e(-\beta N) d \beta+O\left(\frac{N^{3 \gamma-1}}{\log ^{20} N}\right) .
\end{aligned}
$$


Since

$$
\begin{aligned}
\int_{1 /(q Q)}^{1 / 2}\left(\sum_{N / 10<s \leq N} s^{\gamma-1} e(\beta s)\right) & \left(\sum_{N / 10<s \leq N} \frac{s^{\gamma-1} e(\beta s)}{\log s}\right)^{2} e(-\beta N) d \beta \\
& \ll \int_{1 /(q Q)}^{1 / 2} N^{3(\gamma-1)} \frac{d \beta}{\beta^{3}} \ll \frac{q^{2} N^{3 \gamma-1}}{\log ^{160} N},
\end{aligned}
$$

we obtain

$$
\Sigma_{4}=\frac{1}{\gamma} I(N) \sum_{q \leq \log ^{80} N} \frac{\mu^{2}(q) C(q,-N)}{\varphi^{2}(q)} \sum_{\substack{r \sim R \\ q \mid r}} \frac{j(r)}{r}+O\left(\frac{N^{3 \gamma-1}}{\log ^{20} N}\right),
$$

where

$$
I(N)=\sum_{\substack{N=n_{1}+n_{2}+n_{3} \\ N / 10<n_{1}, n_{2}, n_{3} \leq N}} \frac{\gamma^{3}\left(n_{1} n_{2} n_{3}\right)^{\gamma-1}}{\log n_{2} \log n_{3}}
$$

Let

$$
\begin{aligned}
\Omega & =\sum_{q \leq \log ^{80} N} \frac{\mu^{2}(q) C(q,-N)}{\varphi^{2}(q)} \sum_{\substack{r \sim R \\
q \mid r}} \frac{j(r)}{r} \\
& =\sum_{r \sim R} \frac{j(r)}{r} \sum_{\substack{q \leq \log ^{80} \\
q \mid r}} \frac{\mu^{2}(q) C(q,-N)}{\varphi^{2}(q)} .
\end{aligned}
$$

Now,

$$
\sum_{r \sim R} \frac{j(r)}{r} \sum_{\substack{q>\log ^{80} N \\ q \mid r}} \frac{\mu^{2}(q) C(q,-N)}{\varphi^{2}(q)} \ll \frac{1}{\log ^{60} N} \sum_{r \sim R} \frac{d^{2}(r)}{r} \ll \frac{1}{\log ^{50} N},
$$

so that

$$
\begin{aligned}
\Omega & =\sum_{r \sim R} \frac{j(r)}{r} \sum_{q \mid r} \frac{\mu^{2}(q) C(q,-N)}{\varphi^{2}(q)}+O\left(\frac{1}{\log ^{50} N}\right) \\
& =\sum_{r \sim R} \frac{\omega(r) j(r)}{r}+O\left(\frac{1}{\log ^{50} N}\right) \\
& =\gamma \sum_{m \sim M} \sum_{k \sim K} a(m) b(k) \frac{\omega(m k)}{m k}+O\left(\frac{1}{\log ^{50} N}\right) .
\end{aligned}
$$

Hence

$$
\Sigma_{4}=I(N) \sum_{m \sim M} \sum_{k \sim K} a(m) b(k) \frac{\omega(m k)}{m k}+O\left(\frac{N^{3 \gamma-1}}{\log ^{20} N}\right) .
$$


Finally,

$$
\Sigma_{1}=I(N) \sum_{m \sim M} \sum_{k \sim K} a(m) b(k) \frac{\omega(m k)}{m k}+O\left(\frac{N^{3 \gamma-1}}{\log ^{20} N}\right) .
$$

The proof of Lemma 10 is complete.

Lemma 11. Assume that $M, K \ll N^{5 / 16}$ and that $a(m), b(k)=O(1)$. Let $\omega(r)$ be defined in (21). Let

$$
\begin{aligned}
J_{1}(N)= & \sum_{N^{5 / 16}<p_{1} \leq N^{1 / 2}} \frac{1}{p_{1}} \sum_{\substack{N=n_{1}+n_{2}+n_{3} \\
N / 10<n_{1}, n_{2}, n_{3} \leq N}} \frac{\gamma^{3}\left(n_{1} n_{2} n_{3}\right)^{\gamma-1}}{\log \frac{n_{2}}{p_{1}} \log n_{3}}, \\
J_{2}(N)= & \sum_{N^{5 / 16}<p_{1} \leq N^{1 / 3}} \sum_{p_{1}<p_{2}<\sqrt{N / p_{1}}} \frac{1}{p_{1} p_{2}} \\
& \times \sum_{\substack{N=n_{1}+n_{2}+n_{3} \\
N / 10<n_{1}, n_{2}, n_{3} \leq N}} \frac{\gamma^{3}\left(n_{1} n_{2} n_{3}\right)^{\gamma-1}}{\log \frac{n_{2}}{p_{1} p_{2}} \log n_{3}} .
\end{aligned}
$$

Then

$$
\begin{aligned}
& \sum_{m \sim M} \sum_{k \sim K} a(m) b(k)\left(\sum_{\substack{N=m k l+p_{1} p_{2}+p_{3} \\
N / 10<m k l \leq N}} N(m k l) N\left(p_{1} p_{2}\right) N\left(p_{3}\right)\right. \\
& N / 10<p_{1} p_{2}, p_{3} \leq N \\
& N^{5 / 16}<p_{1} \leq N^{1 / 2} \\
& \left.-\frac{\omega(m k)}{m k} J_{1}(N)\right)=O\left(\frac{N^{3 \gamma-1}}{\log ^{20} N}\right)
\end{aligned}
$$

and

$$
\begin{aligned}
& \sum_{m \sim M} \sum_{k \sim K} a(m) b(k)\left(\sum_{\substack{N=m k l+p_{1} p_{2} p_{3}+p_{4} \\
N / 10<m k l \leq N \\
N / 10<p_{1} p_{2} p_{3}, p_{4} \leq N \\
N^{5 / 16}<p_{1} \leq N^{1 / 3} \\
p_{1}<p_{2}<p_{3}}} N(m k l) N\left(p_{1} p_{2} p_{3}\right) N\left(p_{4}\right)\right. \\
& \left.-\frac{\omega(m k)}{m k} J_{2}(N)\right)=O\left(\frac{N^{3 \gamma-1}}{\log ^{20} N}\right) .
\end{aligned}
$$

This can be proved in almost the same way as Lemma 10 . 
4. Sieve method. Assume that

$$
\begin{gathered}
\mathcal{A}=\left\{a: a=N-p_{1}-p_{2}, N(a)=N\left(p_{1}\right)=N\left(p_{2}\right)=1,\right. \\
\left.N / 10<p_{1}, p_{2} \leq N, p_{1}+p_{2}<9 N / 10\right\}, \\
\mathcal{B}=\left\{b: b=N-d-p_{4}, N(b)=N(d)=N\left(p_{4}\right)=1,\right. \\
N / 10<d, p_{4} \leq N, d+p_{4}<9 N / 10, d=p_{1} p_{2}\left(N^{5 / 16}<p_{1} \leq N^{1 / 2},\right. \\
\left.\left.p_{1}<p_{2}\right) \text { or } d=p_{1} p_{2} p_{3}\left(N^{5 / 16}<p_{1} \leq N^{1 / 3}, p_{1}<p_{2}<p_{3}\right)\right\}
\end{gathered}
$$

and that

$$
P(z)=\prod_{p<z} p, \quad \mathcal{S}(\mathcal{A}, z)=\sum_{\substack{a \in \mathcal{A} \\(a, P(z))=1}} 1, \quad \mathcal{S}(\mathcal{B}, w)=\sum_{\substack{b \in \mathcal{B} \\(b, P(w))=1}} 1 .
$$

Note once again that $p \in P_{\gamma}$ is equivalent to $N(p)=1$. Applying Buchstab's identity, we get

$$
\begin{aligned}
T_{1}(N) \geq & \mathcal{S}\left(\mathcal{A}, N^{1 / 2}\right) \\
= & \mathcal{S}\left(\mathcal{A}, N^{3 / 16}\right)-\sum_{N^{3 / 16}<p \leq N^{5 / 16}} \mathcal{S}\left(\mathcal{A}_{p}, p\right) \\
& -\sum_{\substack{N^{5 / 16}<p \leq N^{1 / 2}\\
}} \mathcal{S}\left(\mathcal{A}_{p}, p\right) \\
= & \mathcal{S}_{1}-\mathcal{S}_{2}-\mathcal{S}_{3} .
\end{aligned}
$$

Using Buchstab's identity again, we obtain

$$
\begin{aligned}
\mathcal{S}_{1}= & \mathcal{S}\left(\mathcal{A}, N^{2.5 / 16}\right)-\sum_{N^{2.5 / 16}<p \leq N^{3 / 16}} \mathcal{S}\left(\mathcal{A}_{p}, p\right) \\
= & \mathcal{S}\left(\mathcal{A}, N^{2.5 / 16}\right)-\sum_{N^{2.5 / 16}<p \leq N^{3 / 16}} \mathcal{S}\left(\mathcal{A}_{p},\left(\frac{N^{10 / 16}}{p}\right)^{1 / 5}\right) \\
& +\sum_{N^{2.5 / 16}<p \leq N^{3 / 16}} \mathcal{S}\left(\mathcal{A}_{p q}, q\right) \\
& +\sum_{N^{2.5 / 16}<p \leq N^{3 / 16}} \sum_{N^{5 / 16} / p<q<\left(N^{10 / 16} / p\right)^{1 / 3}} \mathcal{S}\left(\mathcal{A}_{p q}, q\right) \\
& +\sum_{N^{2.5 / 16}<p \leq N^{3 / 16}} \mathcal{S}\left(\mathcal{A}_{p q}, q\right) \\
= & \Phi_{1}-\Phi_{2}+\Phi_{3}+\Phi_{4}+\Phi_{5} .
\end{aligned}
$$

Next,

$(32)$

$$
\mathcal{S}_{3}=\sum_{N^{5 / 16}<p \leq N^{1 / 2}} \mathcal{S}\left(\mathcal{A}_{p}, p\right)
$$




$$
\begin{gathered}
=\#\left\{d: d=N-p_{4}-p_{5}, N(d)=N\left(p_{4}\right)=N\left(p_{5}\right)=1,\right. \\
N / 10<p_{4}, p_{5} \leq N, p_{4}+p_{5}<9 N / 10, \\
d=p_{1} p_{2}\left(N^{5 / 16}<p_{1} \leq N^{1 / 2}, p_{1}<p_{2}\right) \\
\text { or } \left.d=p_{1} p_{2} p_{3}\left(N^{5 / 16}<p_{1} \leq N^{1 / 3}, p_{1}<p_{2}<p_{3}\right)\right\} \\
=\#\left\{p_{4}: p_{4}=N-d-p_{5}, N\left(p_{4}\right)=N(d)=N\left(p_{5}\right)=1,\right. \\
N / 10<d, p_{5} \leq N, d+p_{5}<9 N / 10, \\
d=p_{1} p_{2}\left(N^{5 / 16}<p_{1} \leq N^{1 / 2}, p_{1}<p_{2}\right) \\
\text { or } \left.d=p_{1} p_{2} p_{3}\left(N^{5 / 16}<p_{1} \leq N^{1 / 3}, p_{1}<p_{2}<p_{3}\right)\right\} \\
=\mathcal{S}\left(\mathcal{B}, N^{1 / 2}\right) .
\end{gathered}
$$

Using Buchstab's identity, we have

$$
\begin{aligned}
(33) \mathcal{S}\left(\mathcal{B}, N^{1 / 2}\right)= & \mathcal{S}\left(\mathcal{B}, N^{2.5 / 16}\right)-\sum_{N^{2.5 / 16}<p \leq N^{3 / 16}} \mathcal{S}\left(\mathcal{B}_{p}, p\right) \\
& -\sum_{N^{3 / 16}<p \leq N^{5 / 16}} \mathcal{S}\left(\mathcal{B}_{p}, p\right)-\sum_{N^{5 / 16}<p \leq N^{1 / 2}} \mathcal{S}\left(\mathcal{B}_{p}, p\right) \\
\leq & \mathcal{S}\left(\mathcal{B}, N^{2.5 / 16}\right)-\sum_{N^{2.5 / 16}<p \leq N^{3 / 16}} \mathcal{S}\left(\mathcal{B}_{p},\left(\frac{N^{10 / 16}}{p}\right)^{1 / 5}\right) \\
& +\sum_{N^{2.5 / 16}<p \leq N^{3 / 16}} \mathcal{S}\left(N^{10 / 16} / p\right)^{1 / 5}<q<N^{5 / 16} / p \\
& +\sum_{N^{2.5 / 16}<p \leq N^{3 / 16}} \sum_{N^{5 / 16} / p<q<p} \mathcal{S}\left(\mathcal{B}_{p q}, q\right) \\
& -\sum_{N^{3 / 16}<p \leq N^{5 / 16}} \mathcal{S}\left(\mathcal{B}_{p}, p\right) \\
= & \Gamma_{1}-\Gamma_{2}+\Gamma_{3}+\Gamma_{4}-\Gamma_{5} .
\end{aligned}
$$

LEMma 12.

$$
\Phi_{1}=\mathcal{S}\left(\mathcal{A}, N^{2.5 / 16}\right) \geq 3.515559 Z(\gamma) C(N) \frac{N^{3 \gamma-1}}{\log ^{3} N},
$$

where $C(N)$ is defined in (2) and

$$
Z(\gamma)=\gamma^{3} \int_{1 / 10}^{8 / 10} u_{1}^{\gamma-1} d u_{1} \int_{1 / 10}^{9 / 10-u_{1}} u_{2}^{\gamma-1}\left(1-u_{1}-u_{2}\right)^{\gamma-1} d u_{2} .
$$

Proof. Take

$$
X=I(N)=\sum_{\substack{N=n_{1}+n_{2}+n_{3} \\ N / 10<n_{1}, n_{2}, n_{3} \leq N}} \frac{\gamma^{3}\left(n_{1} n_{2} n_{3}\right)^{\gamma-1}}{\log n_{2} \log n_{3}}
$$


and

$$
r(d)=\# \mathcal{A}_{d}-\frac{\omega(d)}{d} X,
$$

where $\omega(d)$ is defined in (21).

By Theorem 7.11 of [10], we have

$$
W(z)=\prod_{p<z}\left(1-\frac{\omega(p)}{p}\right)=C(N) \frac{e^{-\gamma}}{\log z}\left(1+O\left(\frac{1}{\log z}\right)\right),
$$

where $\gamma$ is Euler's constant.

Let $z=N^{2.5 / 16}, D=N^{10 / 16}$. By Iwaniec's bilinear sieve method (see Theorem 1 in [5]), we obtain

$$
\Phi_{1} \geq \frac{C(N) X}{\log z}\left(f\left(\frac{\log D}{\log z}\right)-O(\varepsilon)\right)-\sum_{m<N^{5 / 16}} \sum_{k<N^{5 / 16}} a(m) b(k) r(m k),
$$

where $f(u)$ is a standard function. In particular,

$$
f(u)= \begin{cases}\frac{2}{u} \log (u-1), & 2 \leq u \leq 4 \\ \frac{2}{u}\left(\log (u-1)+\int_{3}^{u-1} \frac{d t}{t} \int_{2}^{t-1} \frac{\log (s-1)}{s} d s\right), & 4 \leq u \leq 6 .\end{cases}
$$

By Lemma 10, we have

$$
\sum_{m<N^{5 / 16}} \sum_{k<N^{5 / 16}} a(m) b(k) r(m k)=O\left(\frac{N^{3 \gamma-1}}{\log ^{10} N}\right) .
$$

On the other hand,

$$
\begin{aligned}
X & =\frac{(1+O(\varepsilon)) \gamma^{3}}{\log ^{2} N} \sum_{\substack{N=n_{1}+n_{2}+n_{3} \\
N / 10<n_{1}, n_{2}, n_{3} \leq N}}\left(n_{1} n_{2} n_{3}\right)^{\gamma-1} \\
& =\frac{(1+O(\varepsilon)) \gamma^{3}}{\log ^{2} N} \int_{N / 10}^{8 N / 10} t_{1}^{\gamma-1} d t_{1} \int_{N / 10}^{9 N / 10-t_{1}} t_{2}^{\gamma-1}\left(N-t_{1}-t_{2}\right)^{\gamma-1} d t_{2} \\
& =\frac{(1+O(\varepsilon)) \gamma^{3}}{\log ^{2} N} N^{3 \gamma-1} \int_{1 / 10}^{8 / 10} u_{1}^{\gamma-1} d u_{1} \int_{1 / 10}^{9 / 10-u_{1}} u_{2}^{\gamma-1}\left(1-u_{1}-u_{2}\right)^{\gamma-1} d u_{2} .
\end{aligned}
$$

Hence,

$$
\Phi_{1} \geq 3.515559 Z(\gamma) C(N) \frac{N^{3 \gamma-1}}{\log ^{3} N}
$$


LEMMA 13.

$$
\begin{aligned}
\Phi_{2} & =\sum_{N^{2.5 / 16}<p \leq N^{3 / 16}} \mathcal{S}\left(\mathcal{A}_{p},\left(\frac{N^{10 / 16}}{p}\right)^{1 / 5}\right) \\
& \leq 1.130791 Z(\gamma) C(N) \frac{N^{3 \gamma-1}}{\log ^{3} N} .
\end{aligned}
$$

Proof. Take

$$
z(p)=\left(\frac{N^{10 / 16}}{p}\right)^{1 / 5}, \quad D(p)=\frac{N^{10 / 16}}{p} .
$$

By Iwaniec's bilinear sieve method, we obtain

$$
\Phi_{2} \leq(1+O(\varepsilon)) C(N) X \sum_{N^{2.5 / 16}<p \leq N^{3 / 16}} \frac{1}{p \log z(p)} F\left(\frac{\log D(p)}{\log z(p)}\right)+R^{+},
$$

where

$$
R^{+}=\sum_{N^{2.5 / 16}<p \leq N^{3 / 16}} \sum_{h<N^{5 / 16} / p} \sum_{k<N^{5 / 16}} c(h) b(k) r(p h k),
$$

and $F(u)$ is a standard function. In particular,

$$
F(u)= \begin{cases}\frac{2}{u}, & 2 \leq u \leq 3 ; \\ \frac{2}{u}\left(1+\int_{2}^{u-1} \frac{\log (t-1)}{t} d t\right), & 3 \leq u \leq 5 .\end{cases}
$$

In $R^{+}$, let $p h=m$. By Lemma 10, we have

$$
R^{+}=O\left(\frac{N^{3 \gamma-1}}{\log ^{10} N}\right)
$$

From the above discussion and the prime number theorem, we have

$$
\begin{aligned}
\Phi_{2} \leq & Z(\gamma) C(N) \frac{N^{3 \gamma-1}}{\log ^{2} N} \sum_{N^{2.5 / 16}<p \leq N^{3 / 16}} \frac{5 F(5)}{p \log \frac{N^{10 / 16}}{p}}+O\left(\frac{\varepsilon N^{3 \gamma-1}}{\log ^{3} N}\right) \\
= & Z(\gamma) C(N) \frac{N^{3 \gamma-1}}{\log ^{3} N} \int_{2.5 / 16}^{3 / 16} \frac{2 d t}{t\left(\frac{10}{16}-t\right)}\left(1+\int_{2}^{4} \frac{\log (u-1)}{u} d u\right) \\
& +O\left(\frac{\varepsilon N^{3 \gamma-1}}{\log ^{3} N}\right) \\
\leq & 1.130791 Z(\gamma) C(N) \frac{N^{3 \gamma-1}}{\log ^{3} N} .
\end{aligned}
$$


LEMMA 14.

$$
\begin{aligned}
\Phi_{4} & =\sum_{N^{2.5 / 16}<p \leq N^{3 / 16}} \sum_{N^{5 / 16} / p<q<\left(N^{10 / 16} / p\right)^{1 / 3}} \mathcal{S}\left(\mathcal{A}_{p q}, q\right) \\
& \geq 0.011651 Z(\gamma) C(N) \frac{N^{3 \gamma-1}}{\log ^{3} N} .
\end{aligned}
$$

Pro of. Take

$$
D(p, q)=\frac{N^{10 / 16}}{p q} .
$$

By Iwaniec's bilinear sieve method, we have

$$
\begin{aligned}
& \Phi_{4} \geq(1+O(\varepsilon)) C(N) X \sum_{N^{2.5 / 16}<p \leq N^{3 / 16}} \sum_{N^{5 / 16} / p<q<\left(N^{10 / 16 / p)^{1 / 3}}\right.} \frac{1}{p q \log q} \\
& \times f\left(\frac{\log D(p, q)}{\log q}\right)-R^{-},
\end{aligned}
$$

where

$$
\sum_{N^{2.5 / 16}<p \leq N^{3 / 16}} \sum_{N^{5 / 16} / p<q<\left(N^{10 / 16} / p\right)^{1 / 3}} \sum_{h<N^{5 / 16} / p} \sum_{g<N^{5 / 16} / q} c(h) v(g) r(p q h g) .
$$

In $R^{-}$, let $p h=m, q g=k$. By Lemma 10 , we have

$$
R^{-}=O\left(\frac{N^{3 \gamma-1}}{\log ^{10} N}\right)
$$

Hence,

$$
\begin{aligned}
\Phi_{4} \geq & Z(\gamma) C(N) \frac{N^{3 \gamma-1}}{\log ^{3} N} \int_{2.5 / 16}^{3 / 16} \frac{d t}{t} \int_{5 / 16-t}^{(10 / 16-t) / 3} \frac{2}{w\left(\frac{10}{16}-t-w\right)} \\
& \times \log \left(\frac{\left(\frac{10}{16}-t\right)}{w}-2\right) d w+O\left(\frac{\varepsilon N^{3 \gamma-1}}{\log ^{3} N}\right) \\
\geq & 0.011651 Z(\gamma) C(N) \frac{N^{3 \gamma-1}}{\log ^{3} N} .
\end{aligned}
$$

LEMMA 15.

$$
\Gamma_{1}=\mathcal{S}\left(\mathcal{B}, N^{2.5 / 16}\right) \leq 2.926882 Z(\gamma) C(N) \frac{N^{3 \gamma-1}}{\log ^{3} N} .
$$

Proof. We take $Y=J_{1}(N)+J_{2}(N)$, where $J_{1}(N), J_{2}(N)$ are defined in (28) and (29) respectively, and

$$
r(d)=\# \mathcal{B}_{d}-\frac{\omega(d)}{d} Y .
$$


By Iwaniec's bilinear sieve method, we get

$$
\Gamma_{1} \leq \frac{C(N) Y}{\log N} \cdot \frac{16}{2.5}(F(4)+O(\varepsilon))+\sum_{m<N^{5 / 16}} \sum_{k<N^{5 / 16}} a(m) b(k) r(m k) .
$$

Applying Lemma 11, we get

$$
\sum_{m<N^{5 / 16}} \sum_{k<N^{5 / 16}} a(m) b(k) r(m k)=O\left(\frac{N^{3 \gamma-1}}{\log ^{10} N}\right) .
$$

Hence,

Now,

$$
\Gamma_{1} \leq 3.671111 \cdot \frac{C(N) Y}{\log N} .
$$

$$
\begin{aligned}
Y= & \frac{(1+O(\varepsilon)) \gamma^{3}}{\log N} \sum_{\substack{N=n_{1}+n_{2}+n_{3} \\
N / 10<n_{1}, n_{2}, n_{3} \leq N}}\left(n_{1} n_{2} n_{3}\right)^{\gamma-1}\left(\sum_{N^{5 / 16}<p_{1} \leq N^{1 / 2}} \frac{1}{p_{1} \log \frac{N}{p_{1}}}\right. \\
& \left.+\sum_{N^{5 / 16}<p_{1} \leq N^{1 / 3}} \sum_{p_{1}<p_{2}<\sqrt{N / p_{1}}} \frac{1}{p_{1} p_{2} \log \frac{N}{p_{1} p_{2}}}\right) \\
= & (1+O(\varepsilon)) Z(\gamma) \frac{N^{3 \gamma-1}}{\log ^{2} N}\left(\int_{5 / 16}^{1 / 2} \frac{d t}{t(1-t)}+\int_{5 / 16}^{1 / 3} \frac{d t}{t} \int_{t}^{(1-t) / 2} \frac{d w}{w(1-t-w)}\right) \\
\leq & 0.797274 Z(\gamma) \frac{N^{3 \gamma-1}}{\log ^{2} N} .
\end{aligned}
$$

Therefore,

LEMMA 16.

$$
\Gamma_{1} \leq 2.926882 Z(\gamma) C(N) \frac{N^{3 \gamma-1}}{\log ^{3} N}
$$

$$
\begin{aligned}
\Gamma_{2} & =\sum_{N^{2.5 / 16}<p \leq N^{3 / 16}} \mathcal{S}\left(\mathcal{B}_{p},\left(\frac{N^{10 / 16}}{p}\right)^{1 / 5}\right) \\
& \geq 0.898396 Z(\gamma) C(N) \frac{N^{3 \gamma-1}}{\log ^{3} N} .
\end{aligned}
$$

Proof. Using Lemma 11, in almost the same way as in Lemma 13, we obtain

$$
\begin{aligned}
\Gamma_{2} & \geq(1+O(\varepsilon)) C(N) Y \sum_{N^{2.5 / 16}<p \leq N^{3 / 16}} \frac{5 f(5)}{p \log \frac{N^{10 / 16}}{p}} \\
& =(1+O(\varepsilon)) Z(\gamma) C(N) \frac{N^{3 \gamma-1}}{\log ^{3} N} \int_{2.5 / 16}^{3 / 16} \frac{2 d t}{t\left(\frac{10}{16}-t\right)}
\end{aligned}
$$




$$
\begin{aligned}
& \times\left(\log 4+\int_{3}^{4} \frac{d t}{t} \int_{2}^{t-1} \frac{\log (s-1)}{s} d s\right) \\
& \times\left(\int_{5 / 16}^{1 / 2} \frac{d t}{t(1-t)}+\int_{5 / 16}^{1 / 3} \frac{d t}{t} \int_{t}^{(1-t) / 2} \frac{d w}{w(1-t-w)}\right) \\
\geq & 0.898396 Z(\gamma) C(N) \frac{N^{3 \gamma-1}}{\log ^{3} N} .
\end{aligned}
$$

LEMMA 17.

$$
\begin{aligned}
\Gamma_{4} & =\sum_{N^{2.5 / 16}<p \leq N^{3 / 16}} \sum_{N^{5 / 16 / p<q<p}} \mathcal{S}\left(\mathcal{B}_{p q}, q\right) \\
& \leq 0.194188 Z(\gamma) C(N) \frac{N^{3 \gamma-1}}{\log ^{3} N} .
\end{aligned}
$$

Proof. Applying Lemma 11, in almost the same way as in Lemma 14, we get

$$
\begin{aligned}
\Gamma_{4} \leq & \sum_{N^{2.5 / 16}<p \leq N^{3 / 16}} \sum_{N^{5 / 16} / p<q<p} \mathcal{S}\left(\mathcal{B}_{p q},\left(\frac{N^{10 / 16}}{p q}\right)^{1 / 3}\right) \\
\leq & (1+O(\varepsilon)) C(N) Y \sum_{N^{2.5 / 16}<p \leq N^{3 / 16}} \sum_{N^{5 / 16 / p<q<p}} \frac{3 F(3)}{p q \log \frac{N^{10 / 16}}{p q}} \\
= & (1+O(\varepsilon)) Z(\gamma) C(N) \frac{N^{3 \gamma-1}}{\log ^{3} N} \int_{2.5 / 16}^{3 / 16} \frac{d t}{t} \int_{5 / 16-t}^{t} \frac{2 d w}{w\left(\frac{10}{16}-t-w\right)} \\
& \times\left(\int_{5 / 16}^{1 / 2} \frac{d t}{t(1-t)}+\int_{5 / 16}^{1 / 3} \frac{d t}{t} \int_{t}^{(1-t) / 2} \frac{d w}{w(1-t-w)}\right) \\
\leq & 0.194188 Z(\gamma) C(N) \frac{N^{3 \gamma-1}}{\log ^{3} N} .
\end{aligned}
$$

\section{Asymptotic formulas}

Lemma 18. Assume that $N^{11 / 16} \ll M \ll N^{13 / 16}, 0 \leq a(m)=O(1)$ and that $a(m)=0$ if $m$ has a prime factor $<N^{\varepsilon}$. Then

$$
\begin{aligned}
\Sigma= & \sum_{\substack{N=m p_{1}+p_{2}+p_{3} \\
N / 10<m p_{1}, p_{2}, p_{3} \leq N \\
m \sim M}} a(m) N\left(m p_{1}\right) N\left(p_{2}\right) N\left(p_{3}\right) \\
= & (1+O(\varepsilon)) Z(\gamma) C(N) \frac{N^{3 \gamma-1}}{N \log ^{2} N} \sum_{m \sim M} a(m) \sum_{N / m<p \leq 2 N / m} 1 \\
& +O\left(\frac{N^{3 \gamma-1}}{\log ^{10} N}\right) .
\end{aligned}
$$


Proof. We have

$$
\begin{aligned}
\Sigma= & \int_{0}^{1} \sum_{\substack{N / 10<m p_{1} \leq N \\
m \sim M}} a(m) N\left(m p_{1}\right) e\left(\alpha m p_{1}\right) \\
& \times\left(\sum_{N / 10<p \leq N} N(p) e(\alpha p)\right)^{2} e(-\alpha N) d \alpha .
\end{aligned}
$$

Using the same reasoning as in Lemma 10, we get

$$
\Sigma=\int_{E_{1}} g(\alpha) S^{2}(\alpha) e(-\alpha N) d \alpha+O\left(\frac{N^{3 \gamma-1}}{\log ^{10} N}\right),
$$

where $E_{1}$ is defined in Lemma 10 ,

$$
g(\alpha)=\gamma \sum_{\substack{N / 10<m p_{1} \leq N \\ m \sim M}} a(m)\left(m p_{1}\right)^{\gamma-1} e\left(\alpha m p_{1}\right)
$$

and

$$
\mathcal{S}(\alpha)=\gamma \sum_{N / 10<p \leq N} p^{\gamma-1} e(\alpha p) .
$$

Note that if $a(m) \neq 0$ and $q \leq \log ^{80} N$, then $(m, q)=1$. Thus,

$$
g(\alpha)=\gamma \sum_{\substack{l=1 \\(l, q)=1}}^{q} e\left(\frac{a l}{q}\right) \sum_{m \sim M} a(m) m^{\gamma-1} \sum_{\substack{N /(10 m)<p_{1} \leq N / m \\ m p_{1} \equiv l(\bmod q)}} p_{1}^{\gamma-1} e\left(\beta m p_{1}\right) .
$$

Let $\bar{m}$ be a number such that $\bar{m} m \equiv 1(\bmod q)$. Using the discussion in Lemma 10, we have

$$
\begin{aligned}
& \sum_{\substack{N /(10 m)<p_{1} \leq N / m \\
m p_{1} \equiv l(\bmod q)}} p_{1}^{\gamma-1} e\left(\beta m p_{1}\right) \\
= & \sum_{\substack{N /(10 m)<p_{1} \leq N / m \\
p_{1} \equiv \bar{m} l(\bmod q)}} p_{1}^{\gamma-1} e\left(\beta m p_{1}\right)=\int_{N /(10 m)}^{N / m} t^{\gamma-1} e(\beta m t) d(\pi(t ; \bar{m} l, q)) \\
= & \frac{1}{\varphi(q)} \int_{N /(10 m)}^{N / m} \frac{t^{\gamma-1} e(\beta m t)}{\log t} d t+O\left(\left(\frac{N}{m}\right)^{\gamma} \exp (-c \sqrt{\log N})\right) \\
= & \frac{m^{-\gamma}}{\varphi(q)} \int_{N / 10}^{N} \frac{u^{\gamma-1} e(\beta u)}{\log \frac{u}{m}} d u+O\left(\left(\frac{N}{m}\right)^{\gamma} \exp (-c \sqrt{\log N})\right)
\end{aligned}
$$




$$
=\frac{m^{-\gamma}}{\varphi(q)} \sum_{N / 10<s \leq N} \frac{s^{\gamma-1} e(\beta s)}{\log \frac{s}{m}}+O\left(\left(\frac{N}{m}\right)^{\gamma} \exp (-c \sqrt{\log N})\right) .
$$

Therefore,

$$
\begin{aligned}
g(\alpha)= & \gamma \frac{\mu(q)}{\varphi(q)} \sum_{m \sim M} \frac{a(m)}{m} \sum_{N / 10<s \leq N} \frac{s^{\gamma-1} e(\beta s)}{\log \frac{s}{m}} \\
& +O\left(N^{\gamma} \exp \left(-c_{1} \sqrt{\log N}\right)\right),
\end{aligned}
$$

and

$$
\mathcal{S}(\alpha)=\gamma \frac{\mu(q)}{\varphi(q)} \sum_{N / 10<s \leq N} \frac{s^{\gamma-1} e(\beta s)}{\log s}+O\left(N^{\gamma} \exp \left(-c_{2} \sqrt{\log N}\right)\right) .
$$

Hence,

$$
\begin{aligned}
\Sigma= & \sum_{q \leq \log ^{80}} \sum_{\substack{a=0 \\
(a, q)=1}}^{q-1} e\left(-\frac{a N}{q}\right) \int_{-1 /(q Q)}^{1 /(q Q)} g\left(\frac{a}{q}+\beta\right) S^{2}\left(\frac{a}{q}+\beta\right) e(-\beta N) d \beta \\
& +O\left(\frac{N^{3 \gamma-1}}{\log ^{10} N}\right) \\
= & \gamma^{3} \sum_{q \leq \log ^{80} N} \frac{\mu(q) C(q,-N)}{\varphi^{3}(q)} \int_{-1 /(q Q)}^{1 /(q Q)} \sum_{m \sim M} \frac{a(m)}{m} \sum_{N / 10<s \leq N} \frac{s^{\gamma-1} e(\beta s)}{\log \frac{s}{m}} \\
& \left.\times \sum_{N / 10<s \leq N} \frac{s^{\gamma-1} e(\beta s)}{\log s}\right)^{2} e(-\beta N) d \beta+O\left(\frac{N^{3 \gamma-1}}{\log ^{10} N}\right) \\
= & \sum_{q \leq \log ^{80} N} \frac{\mu(q) C(q,-N)}{\varphi^{3}(q)} K(N)+O\left(\frac{N^{3 \gamma-1}}{\log ^{10} N}\right),
\end{aligned}
$$

where

$$
\begin{aligned}
K(N) & =\gamma^{3} \sum_{m \sim M} \frac{a(m)}{m} \sum_{\substack{N=n_{1}+n_{2}+n_{3} \\
N / 10<n_{1}, n_{2}, n_{3} \leq N}} \frac{\left(n_{1} n_{2} n_{3}\right)^{\gamma-1}}{\log \frac{n_{1}}{m} \log n_{2} \log n_{3}} \\
& =(1+O(\varepsilon)) Z(\gamma) \frac{N^{3 \gamma-1}}{\log ^{2} N} \sum_{m \sim M} \frac{a(m)}{m \log \frac{N}{m}} \\
& =(1+O(\varepsilon)) Z(\gamma) \frac{N^{3 \gamma-1}}{N \log ^{2} N} \sum_{m \sim M} a(m) \sum_{N / m<p \leq 2 N / m} 1 .
\end{aligned}
$$


Moreover,

$$
\begin{aligned}
\sum_{q \leq \log ^{80} N} \frac{\mu(q) C(q,-N)}{\varphi^{3}(q)} & =\sum_{q=1}^{\infty} \frac{\mu(q) C(q,-N)}{\varphi^{3}(q)}+O\left(\frac{1}{\log ^{40} N}\right) \\
& =C(N)+O\left(\frac{1}{\log ^{40} N}\right) .
\end{aligned}
$$

The above discussion yields

$$
\begin{aligned}
\Sigma= & (1+O(\varepsilon)) Z(\gamma) C(N) \frac{N^{3 \gamma-1}}{N \log ^{2} N} \sum_{m \sim M} a(m) \sum_{N / m<p \leq 2 N / m} 1 \\
& +O\left(\frac{N^{3 \gamma-1}}{\log ^{10} N}\right) .
\end{aligned}
$$

The proof of Lemma 18 is complete.

Lemma 19.

$$
\mathcal{S}_{2}=\sum_{N^{3 / 16}<p \leq N^{5 / 16}} \mathcal{S}\left(\mathcal{A}_{p}, p\right) \leq 1.198136 Z(\gamma) C(N) \frac{N^{3 \gamma-1}}{\log ^{3} N} .
$$

Proof. From Lemmas 18, 9 and 8, it follows that

$$
\begin{aligned}
\mathcal{S}_{2}= & \sum_{\substack{N=r p+p_{2}+p_{3} \\
N / 10<r p, p_{2}, p_{3} \leq N \\
N^{3 / 16}<p \leq N^{5 / 16} \\
(r, P(p))=1}} N(r p) N\left(p_{2}\right) N\left(p_{3}\right) \\
= & (1+O(\varepsilon)) Z(\gamma) C(N) \frac{N^{3 \gamma-1}}{N \log ^{2} N} \sum_{N^{3 / 16}<p \leq N^{5 / 16}} \sum_{\substack{N / p<r \leq 2 N / p \\
(r, P(p))=1}} 1 \\
& +O\left(\frac{N^{3 \gamma-1}}{\log ^{6} N}\right) \\
= & Z(\gamma) C(N) \frac{N^{3 \gamma-1}}{\log ^{2} N} \sum_{N^{3 / 16}<p \leq N^{5 / 16}} \frac{1}{p \log p} w\left(\frac{\log \frac{N}{p}}{\log p}\right)+O\left(\frac{\varepsilon N^{3 \gamma-1}}{\log ^{3} N}\right) \\
= & Z(\gamma) C(N) \frac{N^{3 \gamma-1}}{\log ^{3} N} \int_{3 / 16}^{5 / 16} \frac{1}{u^{2}} w\left(\frac{1-u}{u}\right) d u+O\left(\frac{\varepsilon N^{3 \gamma-1}}{\log ^{3} N}\right) \\
= & Z(\gamma) C(N) \frac{N^{3 \gamma-1}}{\log ^{3} N} \int_{11 / 5}^{13 / 3} w(t) d t+O\left(\frac{\varepsilon N^{3 \gamma-1}}{\log ^{3} N}\right)
\end{aligned}
$$




$$
\begin{aligned}
\leq & Z(\gamma) C(N) \frac{N^{3 \gamma-1}}{\log ^{3} N}\left(\int_{11 / 5}^{3} \frac{(1+\log (u-1))}{u} d u\right. \\
& \left.+\int_{3}^{4}\left\{\frac{1+\log (u-1)}{u}+\frac{1}{u} \int_{2}^{u-1} \frac{\log (t-1)}{t} d t\right\} d u+0.5644 \int_{4}^{13 / 3} d u\right) \\
\leq & 1.198136 Z(\gamma) C(N) \frac{N^{3 \gamma-1}}{\log ^{3} N} .
\end{aligned}
$$

LEMMA 20.

$$
\begin{aligned}
\Phi_{3} & =\sum_{N^{2.5 / 16}<p \leq N^{3 / 16}} \sum_{\left(N^{10 / 16 / p)^{1 / 5}<q<N^{5 / 16} / p}\right.} \mathcal{S}\left(\mathcal{A}_{p q}, q\right) \\
& \geq 0.399722 Z(\gamma) C(N) \frac{N^{3 \gamma-1}}{\log ^{3} N} .
\end{aligned}
$$

Proof. We have

$$
\Phi_{3}=\sum_{\begin{array}{c}
N=r p q+p_{2}+p_{3} \\
N / 10<r p q, p_{2}, p_{3} \leq N \\
N^{2.5 / 16}<p \leq N^{3 / 16} \\
\left(N^{10 / 16} / p\right)^{1 / 5}<q<N^{5 / 16} / p \\
(r, P(q))=1
\end{array}} N(r p q) N\left(p_{2}\right) N\left(p_{3}\right) .
$$

Note that $N^{3 / 16} \ll p q \ll N^{5 / 16}$ and that $N^{11 / 16} \ll r \ll N^{13 / 16}$. By Lemma 18 with a small modification, and Lemmas 9 and 8 , we have

$$
\begin{aligned}
\Phi_{3}= & (1+O(\varepsilon)) Z(\gamma) C(N) \frac{N^{3 \gamma-1}}{N \log ^{2} N} \\
& \times \sum_{N^{2.5 / 16}<p \leq N^{3 / 16}} \sum_{\left(N^{10 / 16} / p\right)^{1 / 5}<q<N^{5 / 16} / p} \sum_{\substack{N /(p q)<r \leq 2 N /(p q) \\
(r, P(q))=1}} 1 \\
& +O\left(\frac{N^{3 \gamma-1}}{\log ^{6} N}\right) \\
= & Z(\gamma) C(N) \frac{N^{3 \gamma-1}}{\log ^{2} N} \sum_{N^{2.5 / 16}<p \leq N^{3 / 16}} \sum_{\left(N^{10 / 16} / p\right)^{1 / 5}<q<N^{5 / 16} / p} \frac{1}{p q \log q} \\
& \times w\left(\frac{\log \frac{N}{p q}}{\log q}\right)+O\left(\frac{\varepsilon N^{3 \gamma-1}}{\log ^{3} N}\right) \\
\geq & 0.5606 Z(\gamma) C(N) \frac{N^{3 \gamma-1}}{\log ^{3} N} \int_{2.5 / 16}^{3 / 16} \frac{d t}{t} \int_{\substack{(10 / 16-t) / 5 \\
w^{2}}} \frac{d w}{w^{2}} \\
& 0.399722 Z(\gamma) C(N) \frac{N^{3 \gamma-1}}{\log ^{3} N} .
\end{aligned}
$$


Lemma 21. Assume that $N^{11 / 16} \ll M \ll N^{13 / 16}, 0 \leq a(m)=O(1)$ and that $a(m)=0$ if $m$ has a prime factor which is $<N^{\varepsilon}$. Let

$$
\Sigma=\sum_{\substack{N=m p+d+p_{4} \\ N / 10<m p, d, p_{4} \leq N \\ m \sim M}} a(m) N(m p) N(d) N\left(p_{4}\right),
$$

where $d=p_{1} p_{2}\left(N^{5 / 16}<p_{1} \leq N^{1 / 2}, p_{1}<p_{2}\right)$ or $d=p_{1} p_{2} p_{3}\left(N^{5 / 16}<p_{1} \leq\right.$ $\left.N^{1 / 3}, p_{1}<p_{2}<p_{3}\right)$. Then

$$
\begin{aligned}
\Sigma= & (1+O(\varepsilon)) Z(\gamma) C(N) \frac{N^{3 \gamma-1}}{N \log ^{2} N} \sum_{m \sim M} a(m) \sum_{N / m<p \leq 2 N / m} 1 \\
& \times\left(\int_{5 / 16}^{1 / 2} \frac{d t}{t(1-t)}+\int_{5 / 16}^{1 / 3} \frac{d t}{t} \int_{t}^{(1-t) / 2} \frac{d w}{w(1-t-w)}\right)+O\left(\frac{N^{3 \gamma-1}}{\log ^{6} N}\right) .
\end{aligned}
$$

Proof. In almost the same way as in Lemma 18, referring to Lemma 11 , we can get

$$
\begin{aligned}
& \Sigma=(1+O(\varepsilon)) C(N) \sum_{m \sim M} \frac{a(m)}{m} \\
& \times\left(\sum_{N^{5 / 16}<p_{1} \leq N^{1 / 2}} \frac{1}{p_{1}} \sum_{\substack{N=n_{1}+n_{2}+n_{3} \\
N / 10<n_{1}, n_{2}, n_{3} \leq N}} \frac{\gamma^{3}\left(n_{1} n_{2} n_{3}\right)^{\gamma-1}}{\log \frac{n_{1}}{m} \log \frac{n_{2}}{p_{1}} \log n_{3}}\right. \\
& +\sum_{N^{5 / 16}<p_{1} \leq N^{1 / 3}} \sum_{p_{1}<p_{2}<\sqrt{N / p_{1}}} \frac{1}{p_{1} p_{2}} \\
& \left.\times \sum_{\substack{N=n_{1}+n_{2}+n_{3} \\
N / 10<n_{1}, n_{2}, n_{3} \leq N}} \frac{\gamma^{3}\left(n_{1} n_{2} n_{3}\right)^{\gamma-1}}{\log \frac{n_{1}}{m} \log \frac{n_{2}}{p_{1} p_{2}} \log n_{3}}\right)+O\left(\frac{N^{3 \gamma-1}}{\log ^{6} N}\right) \\
& =(1+O(\varepsilon)) \frac{C(N) \gamma^{3}}{\log N} \sum_{m \sim M} \frac{a(m)}{m \log \frac{N}{m}}\left(\sum_{N^{5 / 16}<p_{1} \leq N^{1 / 2}} \frac{1}{p_{1} \log \frac{N}{p_{1}}}\right. \\
& \left.+\sum_{N^{5 / 16}<p_{1} \leq N^{1 / 3}} \sum_{p_{1}<p_{2}<\sqrt{N / p_{1}}} \frac{1}{p_{1} p_{2} \log \frac{N}{p_{1} p_{2}}}\right) \\
& \times \sum_{\substack{N=n_{1}+n_{2}+n_{3} \\
N / 10<n_{1}, n_{2}, n_{3} \leq N}}\left(n_{1} n_{2} n_{3}\right)^{\gamma-1}+O\left(\frac{N^{3 \gamma-1}}{\log ^{6} N}\right) \\
& =(1+O(\varepsilon)) Z(\gamma) C(N) \frac{N^{3 \gamma-1}}{N \log ^{2} N} \sum_{m \sim M} a(m) \sum_{N / m<p \leq 2 N / m} 1
\end{aligned}
$$




$$
\times\left(\int_{5 / 16}^{1 / 2} \frac{d t}{t(1-t)}+\int_{5 / 16}^{1 / 3} \frac{d t}{t} \int_{t}^{(1-t) / 2} \frac{d w}{w(1-t-w)}\right)+O\left(\frac{N^{3 \gamma-1}}{\log ^{6} N}\right) .
$$

LEMMA 22.

$$
\Gamma_{5}=\sum_{N^{3 / 16}<p \leq N^{5 / 16}} \mathcal{S}\left(\mathcal{B}_{p}, p\right) \geq 0.954253 Z(\gamma) C(N) \frac{N^{3 \gamma-1}}{\log ^{3} N} .
$$

Proof. We have

$$
\Gamma_{5}=\sum_{\begin{array}{c}
N=r p+d+p_{4} \\
N / 10<r p, d, p_{4} \leq N \\
N^{3 / 16}<p \leq N^{5 / 16} \\
(r, P(p))=1
\end{array}} N(r p) N(d) N\left(p_{4}\right),
$$

where $d=p_{1} p_{2}\left(N^{5 / 16}<p_{1} \leq N^{1 / 2}, p_{1}<p_{2}\right)$ or $d=p_{1} p_{2} p_{3}\left(N^{5 / 16}<p_{1} \leq\right.$ $\left.N^{1 / 3}, p_{1}<p_{2}<p_{3}\right)$. By Lemmas 21 and 9 , in almost the same way as in Lemma 19, we have

$$
\begin{aligned}
\Gamma_{5}= & Z(\gamma) C(N) \frac{N^{3 \gamma-1}}{\log ^{3} N} \int_{11 / 5}^{13 / 3} w(t) d t \\
& \times\left(\int_{5 / 16}^{1 / 2} \frac{d t}{t(1-t)}+\int_{5 / 16}^{1 / 3} \frac{d t}{t} \int_{t}^{(1-t) / 2} \frac{d w}{w(1-t-w)}\right)+O\left(\frac{\varepsilon N^{3 \gamma-1}}{\log ^{3} N}\right) .
\end{aligned}
$$

Using Lemma 8, we get

$$
\begin{aligned}
\int_{11 / 5}^{13 / 3} w(t) d t \geq & \int_{11 / 5}^{3} \frac{(1+\log (u-1))}{u} d u+\int_{3}^{4}\left\{\frac{1+\log (u-1)}{u}\right. \\
& \left.+\frac{1}{u} \int_{2}^{u-1} \frac{\log (t-1)}{t} d t\right\} d u+0.5607 \int_{4}^{13 / 3} d u
\end{aligned}
$$

$\geq 1.196900$.

Hence

$$
\Gamma_{5} \geq 0.954253 Z(\gamma) C(N) \frac{N^{3 \gamma-1}}{\log ^{3} N}
$$

LEMMA 23.

$$
\begin{aligned}
\Gamma_{3} & =\sum_{N^{2.5 / 16}<p \leq N^{3 / 16}} \sum_{\left(N^{10 / 16 / p)^{1 / 5}<q<N^{5 / 16} / p}\right.} \mathcal{S}\left(\mathcal{B}_{p q}, q\right) \\
& \leq 0.320849 Z(\gamma) C(N) \frac{N^{3 \gamma-1}}{\log ^{3} N} .
\end{aligned}
$$


Proof. We have

$$
\Gamma_{3}=\sum_{\substack{N=r p q+d+p_{4} \\ N / 10<r p q, d, p_{4} \leq N \\ N^{2.5 / 16}<p \leq N^{3 / 16} \\\left(N^{10 / 16} / p\right)^{1 / 5}<q<N^{5 / 16} / p \\(r, P(q))=1}} N(r p q) N(d) N\left(p_{4}\right)
$$

where $d=p_{1} p_{2}\left(N^{5 / 16}<p_{1} \leq N^{1 / 2}, p_{1}<p_{2}\right)$ or $d=p_{1} p_{2} p_{3}\left(N^{5 / 16}<p_{1} \leq\right.$ $\left.N^{1 / 3}, p_{1}<p_{2}<p_{3}\right)$. By Lemma 21 and the deduction in Lemma 20, we get

$$
\begin{aligned}
\Gamma_{3} \leq & 0.5644 Z(\gamma) C(N) \frac{N^{3 \gamma-1}}{\log ^{3} N} \int_{2.5 / 16}^{3 / 16} \frac{d t}{t} \int_{(10 / 16-t) / 5}^{5 / 16-t} \frac{d w}{w^{2}} \\
& \times\left(\int_{5 / 16}^{1 / 2} \frac{d t}{t(1-t)}+\int_{5 / 16}^{1 / 3} \frac{d t}{t} \int_{t}^{(1-t) / 2} \frac{d w}{w(1-t-w)}\right)+O\left(\frac{\varepsilon N^{3 \gamma-1}}{\log ^{3} N}\right) \\
\leq & 0.320849 Z(\gamma) C(N) \frac{N^{3 \gamma-1}}{\log ^{3} N} .
\end{aligned}
$$

6. The proof of the Theorem. Applying Lemmas 12, 13, 20 and 14 to the expression in (31), we get

$$
\mathcal{S}_{1} \geq 2.796141 Z(\gamma) C(N) \frac{N^{3 \gamma-1}}{\log ^{3} N}
$$

Applying Lemmas 15, 16, 23, 17 and 22 to the expression in (33), we obtain

$$
\mathcal{S}_{3} \leq 1.589270 Z(\gamma) C(N) \frac{N^{3 \gamma-1}}{\log ^{3} N} .
$$

In (30), the above two inequalities and Lemma 19 yield

$$
T_{1}(N) \geq 0.008734 Z(\gamma) C(N) \frac{N^{3 \gamma-1}}{\log ^{3} N} \geq \frac{\varrho_{0} C(N) N^{3 \gamma-1}}{\log ^{3} N} .
$$

Hence, the Theorem follows.

\section{References}

[1] A. Balog and J. Friedlander, A hybrid of theorems of Vinogradov and PiatetskiShapiro, Pacific J. Math. 156 (1992), 45-62.

[2] H. Davenport, Multiplicative Number Theory, 2nd ed., Springer, New York, 1980.

[3] G. Harman, On the distribution of $\alpha p$ modulo one, J. London Math. Soc. (2) 27 (1983), 9-18.

[4] D. R. Heath-Brown, The Pjateckiǔ-S̆apiro prime number theorem, J. Number Theory 16 (1983), 242-266. 
[5] H. Iwaniec, A new form of the error term in the linear sieve, Acta Arith. 37 (1980), 307-320.

[6] C. Jia, On Pjateckiǔ-Šapiro prime number theorem (II), Science in China Ser. A 36 (1993), 913-926.

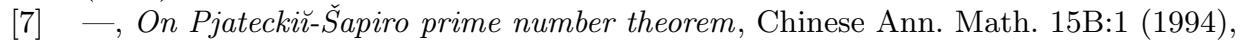
9-22.

[8] G. Kolesnik, Primes of the form $\left[n^{c}\right]$, Pacific J. Math. 118 (1985), 437-447.

[9] H. Q. Liu and J. Rivat, On the Pjateckiǔ-Šapiro prime number theorem, Bull. London Math. Soc. 24 (1992), 143-147.

[10] Chengdong Pan and Chengbiao P an, Goldbach Conjecture, Science Press, Beijing, 1992.

[11] I. I. Piatetski-Shapiro, On the distribution of prime numbers in sequences of the form $[f(n)]$, Mat. Sb. 33 (1953), 559-566 (in Russian).

[12] P. Shiu, A Brun-Titchmarsh theorem for multiplicative functions, J. Reine Angew. Math. 313 (1980), 161-170.

[13] E. Wirsing, Thin subbases, Analysis 6 (1986), 285-308.

INSTITUTE OF MATHEMATICS

ACADEMIA SINICA

BEIJING, 100080, CHINA 